\section{BRAZIULIAN JOURNAL}

OF MEDICAL AND BIOLOGICAL RESFARCH

www.bjournal.com.br
ISSN 0100-879X

Volume 44 (7) 606-728 July 2011

BIOMEDICAL SCIENCES

AND

CLINICAL INVESTIGATION

Braz J Med Biol Res, July 2011, Volume 44(7) 642-646

doi: 10.1590/S0100-879X2011007500072

Inhibition of cytohesin-1 by siRNA leads to reduced IGFR signaling in prostate cancer

Weizhong Zhang, Shuohui Gao, Hanjiao Qin, Yuhong Man, Xiaohua Yang, Jian Cao and Lisen Li

The Brazilian Journal of Medical and Biological Research is partially financed by

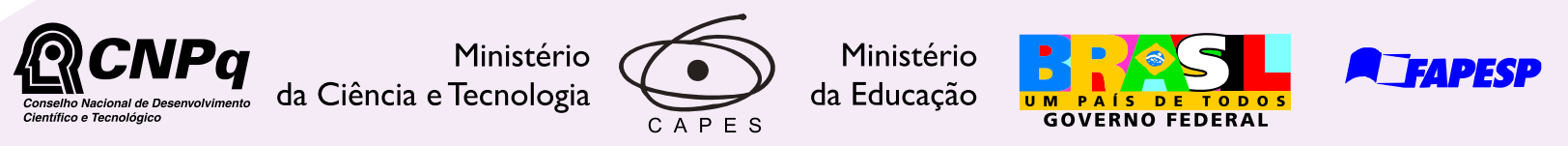

Institutional Sponsors

scie/
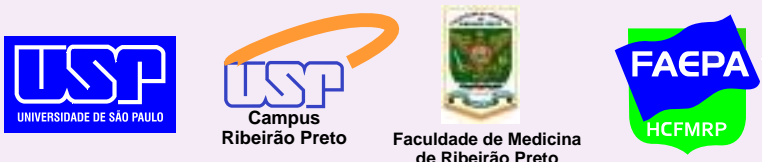


\title{
Inhibition of cytohesin-1 by siRNA leads to reduced IGFR signaling in prostate cancer
}

\author{
Weizhong Zhang, Shuohui Gao, Hanjiao Qin, Yuhong Man, Xiaohua Yang, \\ Jian Cao and Lisen Li \\ Department of Hand Surgery, China-Japan Union Hospital, \\ Jilin University, Changchun, China
}

\begin{abstract}
To explore how cytohesin-1 (CYTH-1) small interfering RNA (siRNA) influences the insulin-like growth factor receptor (IGFR)associated signal transduction in prostate cancer, we transfected human prostate cancer PC-3 cell lines with liposome-encapsulated CYTH-1 siRNA in serum-free medium and exposed the cells to $100 \mathrm{nM}$ IGF-1. The mRNA and protein levels of the signal molecules involved in the IGFR signaling pathways were determined by real-time PCR and detected by Western blotting. The relative mRNA levels of CYTH-1, c-Myc, cyclinD1, and IGF-1R (CYTH-1 siRNA group vs scrambled siRNA group) were 0.26 vs $0.97,0.34$ vs $1.06,0.10$ vs 0.95 , and 0.27 vs $0.41(\mathrm{P}<0.05$ for all), respectively. The relative protein levels of $\mathrm{CYTH}-1$, pIGF-1R, pIRS1, pAkt1, pErk1, c-Myc, and cyclinD1 (CYTH-1 siRNA group vs scrambled siRNA group) were 0.10 vs 1.00 (30 $\mathrm{min}$ ), 0.10 vs 0.98 (30 min), 0.04 vs 0.50 (30 min), 0.10 vs 1.00 (30 min), 0.10 vs 1.00 (30 min), 0.13 vs 0.85 (5 h), and 0.08 vs $0.80(7 \mathrm{~h})$, respectively. The tyrosine kinase activity of IGF-1R was associated with CYTH-1. The proliferative activity of PC-3 cells transfected with CYTH-1 siRNA was significantly lower than that of cells transfected with scrambled siRNA at $48 \mathrm{~h}$ (40.5 vs $87.6 \%, \mathrm{P}<0.05)$ and at $72 \mathrm{~h}(34.5$ vs $93.5 \%, \mathrm{P}<0.05)$. In conclusion, the interference of siRNA with cytohesin-1 leads to reduced IGFR signaling in prostate cancer; therefore, CYTH-1 might serve as a new molecular target for the treatment of prostate cancer
\end{abstract}

Key words: Cytohesins; Insulin-like growth factor 1 receptor; Prostate cancer; Signal transduction; siRNA interference

\section{Introduction}

Insulin-like growth factor-1 (IGF-1) and its receptor (IGF-1R) are involved in the apoptosis, transformation, invasive growth, and distant metastasis of tumor cells (1). Blocking the IGF-1R-associated signal transduction will restore the cellular phenotype, promote apoptosis, increase sensitivity to chemotherapeutic drug, and reduce a tumor cell's tendency to metastasize $(2,3)$. The binding of IGF-1 to IGF-1R plays an important biological role in the activation of downstream signaling pathways and in the genesis, development and metastasis of prostate cancer (4-7).

Cytohesins (CYTHs) are a group of ADP ribosylation factor (ARF) guanosine triphosphatase guanine nucleotide exchange factors that have recently become known to be important regulators of signal transduction in vertebrate and invertebrate biology. They function to regulate the reassembly of the cytoskeleton and the activation of integrin or the integrin signaling system (8). Hafner et al. (9) used a CYTH analogue, SecinH3, to inhibit CYTH gene expres- sion in human liver cells and in a mouse model. The results showed that the insulin receptor complex-associated CYTHs were required by the insulin signaling system in liver cells and that SecinH3 inhibited CYTH-induced insulin resistance in liver cells.

There is an apparent correlation between IGF and insulin and IGFRs and insulin receptors (IR) share the same signaling pathway. Therefore, CYTHs are likely to play an important role in IGF-1R signal transduction.

\section{Material and Methods}

\section{Reagents}

CYTH-1 siRNA and scrambled siRNA were purchased from Santa Cruz Biotechnology (USA); lipofectamine 2000, IGF-1, the total protein extraction kit and the Trizol total RNA extraction kit were purchased from Invitrogen (USA). Anti-CYTH-1 antibody (ab56511), anti-IGF-1R (phospho 
Y1161) antibody (ab39398), anti-IRS1 (phospho Y612) antibody (ab66153), anti-Akt1 (phospho T308) antibody (ab66134), anti-p44MAPK (pErk1, phospho T185 + Y187) antibody [MAPK-YT] (ab50011), anti-c-Myc antibody (ab32), anti-cyclinD1 antibody (ab10540), anti-glyceraldehyde 3-phosphate dehydrogenase (GAPDH) antibody (ab8245), fluorescent-labeled goat anti-mouse secondary antibody, and horseradish peroxidase (HRP)-labeled goat anti-mouse secondary antibody were purchased from Abcam (China); these antibodies were monoclonal. 3-(4,5-dimethylthiazol2-yl)2,5-diphenyltetrazolium bromide (MTT) was purchased from Sigma (USA). The Moloney murine leukemia virus reverse transcriptase (M-MLV RTase) kit was purchased from Promega Corporation (China). The 2X SYBR real-time PCR kit was purchased from Roche (China). The bicinchoninic acid (BCA) protein detection kit and enhanced chemiluminescence $(E C L)$ detection kit were purchased from Pierce Chemicals, Thermo Fisher Scientific Inc. (USA).

\section{Cell lines}

Human prostate cancer PC-3 cell lines, which express prostate-specific antigens (PSAs), IGF-1R and IRS1 (1012), were a gift from Hongyan Lee, Vice-Professor, School of Basic Medicine, Jilin University, China. Cells were cultured in Dulbecco's modified Eagle's medium (DMEM) containing $10 \%$ fetal calf serum (Invitrogen Gibco, USA) in a $5 \% \mathrm{CO}_{2}$ incubator and passaged with $0.25 \%$ trypsin (Sigma) in 0.2 $\mathrm{M}$ phosphate-buffered saline (PBS).

\section{Screening of cytohesins}

PC-3 cells were harvested and total RNA was extracted by the Trizol method according to manufacturer instructions. cDNA was synthesized according to manufacturer instructions. The CYTH-1, CYTH-2 and CYTH-3 genes were amplified and total protein was extracted. The procedures are described in detail below.

\section{Transfection and detection}

PC-3 cells were inoculated into each well of 6-well plates at a concentration of $2 \times 10^{5}$ cells $/ \mathrm{mL}$ in $1 \mathrm{~mL}$ DMEM containing $10 \%$ calf serum and incubated for $24 \mathrm{~h}$. The medium was then refreshed with $200 \mu \mathrm{L}$ serum-free medium. Simultaneously, in accordance with manufacturer instructions, compounds for transfection were prepared by mixing $5 \mu \mathrm{L}$ lipofectamin 2000 and $8 \mu \mathrm{L} 10 \mathrm{nM} \mathrm{CYTH-1}$ siRNA together and diluting in $100 \mu \mathrm{L}$ serum-free DMEM. The compounds were left to stand at room temperature for $20 \mathrm{~min}$. Transfection was performed by adding the compounds to the PC-3 cells and culturing in serum-free medium for $48 \mathrm{~h}$.

Stimulation was performed with IGF-1 (the final concentration was $100 \mathrm{nM}$ ) for $10 \mathrm{~min}$ or $30 \mathrm{~min}$, followed by Western blotting to detect the protein expression of CYTH-1, tyrosine phospho-IGF-1R (pIGF-1R), tyrosine phospho-IRS1 (pIRS1), threonine phospho-Akt1 (pAkt1), and threonine-tyrosine phospho-Erk1 (pErk1). Simultaneously, stimulation was performed with IGF-1 for $30 \mathrm{~min}, 1$, $3,5,7$, or $9 \mathrm{~h}$; real-time PCR and Western blotting were performed to detect the mRNA and protein expression of c-Myc and cyclinD1, respectively. Subsequent cell culturing was performed for 24,48 , or $72 \mathrm{~h}$. Cell proliferation was then detected by the MTT assay. Non-transfected PC-3 cells treated with IGF-1 alone and PC-3 cells treated with both IGF-1 and scrambled siRNA were used as positive controls and negative controls, respectively.

\section{Quantitative PCR (qPCR)}

The PC-3 cells were harvested and total RNA was extracted with the total RNA extract kit using the Trizol method. The first strand cDNA was synthesized using M-MLV RTase according to manufacturer instructions and real-time PCR was performed using the cDNA template according to manufacturer instructions. Amplification of GAPDH was used as an inner control in each reaction system. The reaction conditions were 40 cycles of $95^{\circ} \mathrm{C}$ for $30 \mathrm{~s}, 58^{\circ} \mathrm{C}$ for $60 \mathrm{~s}$, and $72^{\circ} \mathrm{C}$ for $60 \mathrm{~s}$. The primers were designed based on the Genbank sequence using Beacon Designer 7 and the primer sequences were verified by Blast. Primer synthesis and DNA sequencing were performed by Shanghai Sangon (China). The primer sequences were as follows: CYTH1: sense 5'-CTGTGAGGAAGGTTATCGG-3', antisense 5'-TCCAGAGTAGTCCAGTTAGG-3'; CYTH-2: sense 5'-TGTGGTCTTGGAGGTGGAGTC-3', antisense 5'-GG CTGCTGCTGCTTCTGG-3'; CYTH-3: sense 5'-GGAG AAGCAGCAGGAAGG-3', antisense 5'-TCTAACTCAGC ACCACAGC-3'; c-Myc: sense 5'-TGTCCGTCCAAGCAG AGG-3', antisense 5'-CGCACAAGAGTTCCGTAGC-3'; cyclinD1: sense 5'-ACGGCTCACGCTTACCTC-3', antisense 5'-CCCAGACCCTCAGACTTGC-3'; GAPDH (housekeeper gene): sense 5'-AATGTGTCCGTCGTGGATCTG-3', antisense 5'-CAACCTGGTCCTCAGTGTAGC-3'.

\section{Western blotting}

The PC-3 cells were harvested and cell lysis was performed using the eukaryotic cell lysis buffer according to manufacturer instructions, followed by extraction of total protein according to manufacturer instructions. Protein quantity was determined by a BCA method. Using $30 \mu \mathrm{g}$ for each sample, proteins were separated by $12 \%$ SDSPAGE and were blotted with a wet transfer device (BioRad Laboratories, Inc., China) to a nitrocellulose membrane. The membrane was then immersed in blocking solution containing $10 \%$ skim milk in PBS Tween-20 (PBST), followed by shaking for $1 \mathrm{~h}$. After washing three times with Tris-buffered saline Tween-20 (TBST) for 5 min each time, the membrane was immersed in primary antibody diluted $1: 1000$ in the blocking solution at room temperature and then shaken for $1 \mathrm{~h}$. After washing again, the membrane was incubated in HRP-labeled secondary antibody diluted $1: 10,000$ in blocking solution at room temperature and 
then shaken for $1 \mathrm{~h}$. After another rinse, the membrane underwent color development by an ECL method, followed by X-film photography. GAPDH protein was used as an inner control. The gray scale values (total raw density) of blots were measured with the VisionWorksLS analysis software available in the UVP EC3 (600) Imaging System (UVP, LLC, USA).

\section{MTT assay}

Each well containing cells was supplemented with 20 $\mu \mathrm{L}$ MTT solution ( $5 \mathrm{mg} / \mathrm{mL}$ ), followed by incubation in a $\mathrm{CO}_{2}$ incubator for $4 \mathrm{~h}$. The supernatant was discarded and each well was supplemented with $100 \mu \mathrm{L}$ dimethylsulfoxide (DMSO, Sigma, USA). When the purple crystals at the bottom of the well were completely dissolved, the absorbance value was measured with a Thermo Multiskan MK3 microplate reader with a wavelength of $\lambda=490 \mathrm{~nm}$. Cell viability $\%=$ experimental absorbance/normal absorbance $\times 100 \%$.
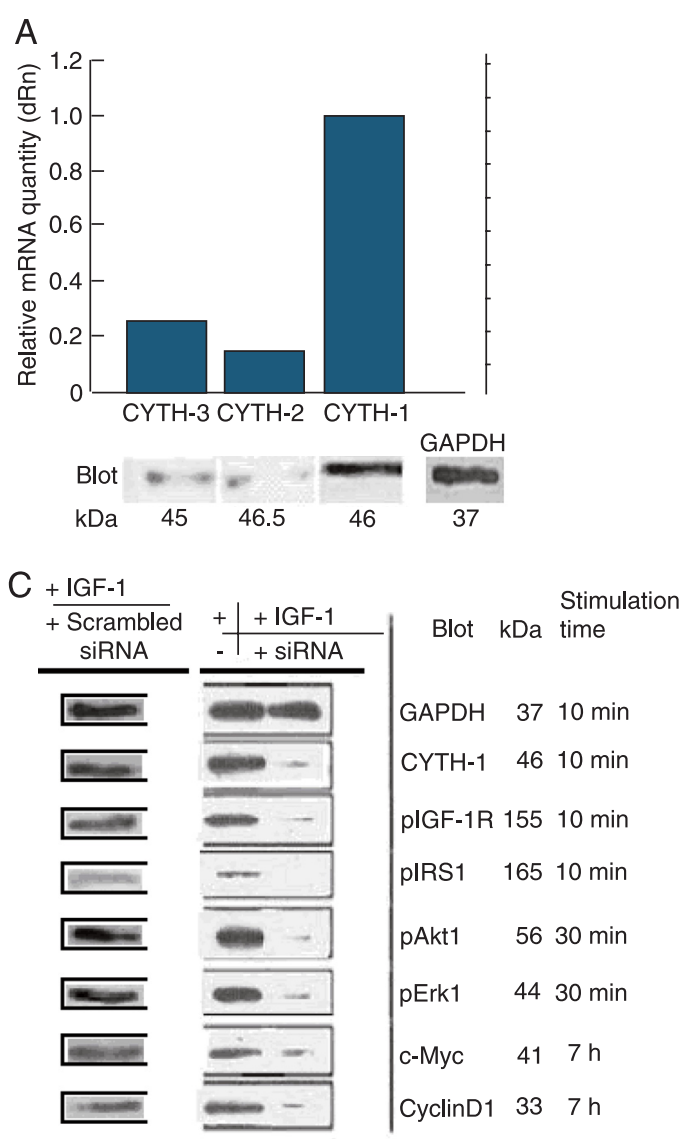

\section{Statistical analysis}

Data are reported as means \pm SD. The statistical software SPSS12.0 was used for statistical analysis. Paired comparisons were performed by the Student $t$-test. $P<0.05$ indicated a statistically significant difference.

\section{Results}

\section{Screening of cytohesins}

Figure 1A shows the expressions of the CYTH-1, CYTH-2 and CYTH-3 genes in the PC-3 cells. The qPCR results showed that the level of $C Y T H-1$ mRNA in the cells was $100 \%$, while the levels of CYTH-2 mRNA and CYTH-3 mRNA were 23 and 18\%, respectively. Because the Western blotting results showed high $\mathrm{CYTH}-1$ protein expression [the gray scale ratio of $\mathrm{CYTH}-1$ to GAPDH was 170/174 (97.7\%)], whereas the CYTH-2 and CYTH-3 proteins were almost undetectable [the gray scale ratios of CYTH-2 and CYTH-3 to GAPDH were 36/174 (20.7\%)

B

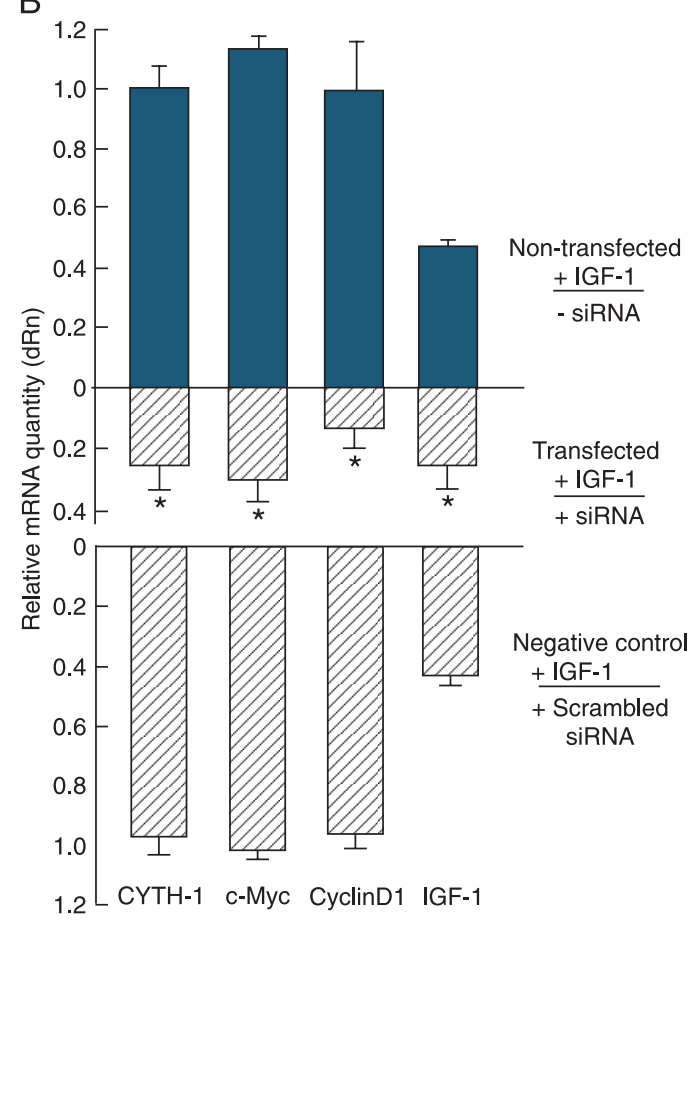

Figure 1. Detection by quantitative PCR (qPCR) and Western blotting. $A$, mRNA and protein levels of CYTH-1, CYTH-2 and CYTH-3 in insulin growth factor-1 (IGF-1)-stimulated PC-3 cells; $B, C$, mRNA and protein levels of related signal molecules in manipulated PC-3 cells. GAPDH was used as a housekeeper gene. + IGF-1 = cells were stimulated by exposure to IGF-1 $(100 \mathrm{nM}) ;+$ siRNA = cells were transfected with $C Y T H-1$ siRNA; -siRNA = cells were not transfected; +scrambled siRNA = cells were transfected with scrambled siRNA. siRNA = small interfering RNA. ${ }^{*} P<0.05$ vs cells transfected with scrambled siRNA $(\mathrm{N}=3$; paired Student $t$-test). 
and $26 / 174$ (14.9\%), respectively], CYTH-1 was used as a target molecule in the subsequent experiments.

\section{Levels of signaling molecules}

Figure $1 \mathrm{~B}$ and $\mathrm{C}$ show the expressions of the cell signaling molecules detected by qPCR and Western blotting. Table 1 shows the complete gray scales of the blots shown in Figure $1 \mathrm{C}$ to present the total levels of detected proteins. Molecular expression and phosphorylation levels in PC-3 cells transfected with $C Y T H-1$ siRNA were lower than in $P C-3$ cells transfected with scrambled siRNA $(P<0.05$ for the mRNA levels). The mRNA levels of CYTH-1, c-Myc, cyclinD1, and IGF-1R (CYTH-1 siRNA group vs scrambled siRNA group) were $0.26 \pm 0.05$ vs $0.97 \pm 0.02,0.34 \pm 0.04$

Table 1. The gray scale values (total raw density) of the blots indicating the total protein levels of detected proteins.

\begin{tabular}{crrl}
\hline $\begin{array}{c}\text { +IGF-1/ } \\
\text { +scrambled siRNA }\end{array}$ & $\begin{array}{c}\text { +IGF-1/ } \\
\text {-siRNA }\end{array}$ & $\begin{array}{r}\text { +IGF-1/ } \\
\text { +siRNA }\end{array}$ & Blot \\
\hline 114.30 & 107.26 & 104.80 & GAPDH \\
114.11 & 112.52 & 10.50 & CYTH-1 \\
112.01 & 113.82 & 10.47 & pIGF-1R \\
57.15 & 55.62 & 4.19 & pIRS1 \\
114.61 & 115.03 & 10.53 & pAkt1 \\
114.19 & 113.54 & 10.58 & pErk1 \\
97.16 & 97.28 & 13.62 & c-Myc \\
91.44 & 90.32 & 8.38 & CyclinD1 \\
\hline
\end{tabular}

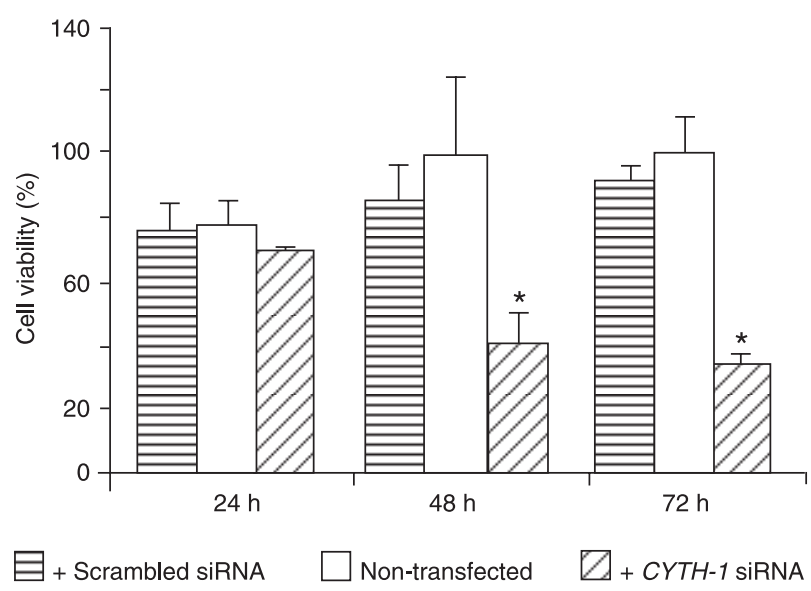

Figure 2. Cell viability of insulin growth factor-1 (IGF-1)-stimulated PC-3 cells transfected with $\mathrm{CYTH}-1$ small interfering RNA (siRNA), transfected with scrambled siRNA and non-transfected. ${ }^{*} \mathrm{P}<0.05$ vs cells transfected with scrambled $\operatorname{siRNA}(\mathrm{N}=4$; paired Student $t$-test). vs $1.06 \pm 0.01,0.10 \pm 0.05$ vs $0.95 \pm 0.02$, and $0.27 \pm 0.05$ vs $0.41 \pm 0.01(\mathrm{P}<0.05$ for all; Figure $1 \mathrm{~B})$, respectively. The protein levels of CYTH-1, pIGF-1R, pIRS1, pAkt1, pErk1, c-Myc, and cyclinD1 (CYTH-1 siRNA group vs scrambled siRNA group) were 0.10 vs 1.00 (30 min), 0.10 vs 0.98 (30 $\mathrm{min}$ ), 0.04 vs 0.50 (30 $\mathrm{min}), 0.10$ vs 1.00 (30 min), 0.10 vs 1.00 (30 min), 0.13 vs 0.85 (5 h), and 0.08 vs 0.80 (7 h), respectively (Figure $1 \mathrm{C}$, Table 1 ). The minimum mRNA level and the minimum protein level for both c-Myc and cyclinD1 appeared at $5 \mathrm{~h}$ (qPCR) and $7 \mathrm{~h}$ (Western blot) after IGF-1 stimulation.

\section{Cell proliferation}

Figure 2 illustrates cell viability. The proliferative activity of PC-3 cells transfected with CYTH-1 siRNA was decreased at 24,48 and $72 \mathrm{~h}$, and was significantly lower than that of cells transfected with scrambled siRNA at $48 \mathrm{~h}(40.5 \pm 8.2$ vs $87.6 \pm 9.2 \%, \mathrm{P}<0.05)$ and at $72 \mathrm{~h}(34.5 \pm 4.2$ vs 93.5 $\pm 5.2 \%, P<0.05)$.

\section{Discussion}

The IGFR signaling system involves complex molecular phosphorylation and includes many molecules for signal transduction, such as IGF-1R, IRS1, Akt1, and Erk1, which can also influence other functional cellular proteins, such as c-Myc and cyclinD1 (10). In the present study, to explore the activity of CYTH-1 and its association with the IGF-1R signaling system in prostate cancer, we used $\mathrm{CYTH}-1$ siRNA to interfere with $\mathrm{CYTH}-1$ expression in $\mathrm{PC}-3$ cells and used qPCR technology and phosphorylated protein-specific antibodies to detect the corresponding changes in the series of IGF-1R signaling molecules mentioned above.

With IGF-1 exposure, PC-3 cells revealed an increase in CYTH-1 mRNA and protein levels. IGF-1 stimulation also increased phosphorylation of IGR-1R at tyrosine 1161 and then pIGF-1R revealed its tyrosine kinase activity to phosphorylate IRS1 at tyrosine 612. Then, the signals were transduced to Akt1 and Erk1 and there was an increase in phosphorylation for both Akt1 (threonine 308 phosphorylation) and Erk1 (threonine 185 and tyrosine 187 phosphorylation). By contrast, the use of CYTH-1 siRNA caused a decrease in CYTH-1 mRNA and protein levels, as well as a decrease in tyrosine 1161 phosphorylation of IGF-1R. The tyrosine kinase activity of IGF-1R also caused a decrease in tyrosine 612 phosphorylation of IRS1 (13). This finding demonstrated that the tyrosine kinase activity of IGF-1R was associated with CYTH-1. Additionally, Akt1 (threonine 308 phosphorylation) and Erk1 (threonine 185 and tyrosine 187 phosphorylation) were decreased, indicating that the signal amplification and transduction pathways were inhibited in an efficient manner $(9,13)$. Hafner et al. (9) reported that phosphorylation of IGF-1R, IRS1, Akt1, and p44MAPK was significantly reduced in liver cells by Secinh3 inhibiting CYTH-1. 
c-Myc is related to tumor transformation (14) and cyclinD1 overexpression can shorten the G1 phase of the cell cycle, reduce cell size and reduce dependence on a mitogen (15). In the present study, the qPCR and Western blotting results showed that both c-Myc and cyclinD1 overexpression were apparently inhibited and the MTT assay results showed that the growth and proliferation of tumor cells were significantly inhibited by $\mathrm{CYTH}-1$ siRNA interference.

In addition, the experimental results showed that the IGFR signaling pathway was not completely suppressed and that a certain degree of cell growth and proliferation remained in the prostate cancer cells. Two possible explana-

\section{References}

1. LeRoith D, Roberts CT Jr. The insulin-like growth factor system and cancer. Cancer Lett 2003; 195: 127-137.

2. Smith GD, Gunnell D, Holly J. Cancer and insulin-like growth factor-I. A potential mechanism linking the environment with cancer risk. BMJ 2000; 321: 847-848.

3. Jones HE, Goddard L, Gee JM, Hiscox S, Rubini M, Barrow $D$, et al. Insulin-like growth factor-I receptor signalling and acquired resistance to gefitinib (ZD1839; Iressa) in human breast and prostate cancer cells. Endocr Relat Cancer 2004; 11: 793-814.

4. LeRoith D, Werner H, Beitner-Johnson D, Roberts CT Jr. Molecular and cellular aspects of the insulin-like growth factor I receptor. Endocr Rev 1995; 16: 143-163.

5. Hellawell GO, Turner GD, Davies DR, Poulsom R, Brewster SF, Macaulay VM. Expression of the type 1 insulin-like growth factor receptor is up-regulated in primary prostate cancer and commonly persists in metastatic disease. Cancer Res 2002; 62: 2942-2950.

6. Djavan B, Waldert M, Seitz C, Marberger M. Insulin-like growth factors and prostate cancer. World J Urol 2001; 19: 225-233.

7. Kojima S, Inahara M, Suzuki H, Ichikawa T, Furuya Y. Implications of insulin-like growth factor-I for prostate cancer therapies. Int J Urol 2009; 16: 161-167. tions are: 1) only CYTH-1 is inhibited in this study, so that CYTH-2 and CYTH-3 may play a role in tumor promotion even though their expression was weak; 2) some inherent bypass signaling pathways may function to rescue the tumor cells, leading to the incomplete suppression of the IGFR signaling pathway.

In conclusion, CYTH-1 plays an important role in the IGFR signaling pathway in prostate cancer cells and suppression of CYTH-1 can reduce the tumor-promoting role of IGFR signaling. Therefore, CYTH-1 might be a new target for treatment of prostate cancer.

8. Kolanus W. Guanine nucleotide exchange factors of the cytohesin family and their roles in signal transduction. Immunol Rev 2007; 218: 102-113.

9. Hafner M, Schmitz A, Grune I, Srivatsan SG, Paul B, Kolanus $W$, et al. Inhibition of cytohesins by SecinH3 leads to hepatic insulin resistance. Nature 2006; 444: 941-944.

10. Kaighn ME, Narayan KS, Ohnuki Y, Lechner JF, Jones LW. Establishment and characterization of a human prostatic carcinoma cell line (PC-3). Invest Urol 1979; 17: 16-23.

11. Chen TR. Chromosome identity of human prostate cancer cell lines, PC-3 and PPC-1. Cytogenet Cell Genet 1993; 62: 183-184.

12. Ohnuki Y, Marnell MM, Babcock MS, Lechner JF, Kaighn ME. Chromosomal analysis of human prostatic adenocarcinoma cell lines. Cancer Res 1980; 40: 524-534.

13. Dey BR, Frick K, Lopaczynski W, Nissley SP, Furlanetto RW. Evidence for the direct interaction of the insulin-like growth factor I receptor with IRS-1, Shc, and Grb10. Mol Endocrinol 1996; 10: 631-641.

14. Schmidt EV. The role of c-myc in cellular growth control. Oncogene 1999; 18: 2988-2996.

15. Alao JP. The regulation of cyclin D1 degradation: roles in cancer development and the potential for therapeutic invention. Mol Cancer 2007; 6: 24. 